\title{
Head and neck cancer and immunotherapy: current knowledge and perspective
}

\author{
Thibault Gauduchon, Thibaut Reverdy, Max Gau, Andy Karabajakian, Laetitia Collet, Eve-Marie Neidhardt, \\ Jérôme Fayette
}

Université Claude Bernard LYON 1, Centre de lutte contre le cancer Léon-Bérard, Département d'oncologie médicale, Lyon 69008, France.

Correspondence to: Dr. Thibault Gauduchon, Université Claude Bernard LYON 1, Centre de lutte contre le cancer Léon-Bérard, Département d'oncologie médicale, Lyon 69008, France. E-mail: thibault.gauduchon@lyon.unicancer.fr

\begin{abstract}
How to cite this article: Gauduchon T, Reverdy T, Gau M, Karabajakian A, Collet L, Neidhardt EM, Fayette J. Head and neck cancer and immunotherapy: current knowledge and perspective. J Cancer Metastasis Treat 2019;5:72.
\end{abstract}

http://dx.doi.org/10.20517/2394-4722.2019.023

Received: 15 Aug 2019 First Decision: 18 Sep 2019 Revised: 16 Oct 2019 Accepted: 16 Oct 2019 Published: 30 Oct 2019

Science Editor: Pravin D. Potdar Copy Editor: Cai-Hong Wang Production Editor: Jing Yu

\begin{abstract}
As with many types of cancer, immunotherapy is changing the management of squamous cell carcinoma of the head and neck (HNSCC). In a locally advanced or metastatic setting, treatment options have long been curtailed, but this paradigm is currently changing. Checkpoint inhibitors were the first to be validated in second-line treatment with PD-1 and PD-L1 inhibitors and these treatments are available in USA for first-line use. In addition, many studies are underway to use its molecules earlier in the care or try to increase their effectiveness with associations. The issues of patient selection that would benefit the most from immunotherapy and the evaluation of the response to these treatments are not completely solved. The goal is here to update the possibilities of current treatment by immunotherapy in HNSCC as well as on various development pathways in progress.
\end{abstract}

Keywords: Head and neck squamous cell carcinoma, checkpoint inhibitors, immunotherapy, clinical trial

\section{INTRODUCTION}

Head and neck cancers are currently responsible for more than 700,000 cases and more than 350,000 deaths per year ${ }^{[1]}$. After a heavy treatment combining surgery, radiotherapy and chemotherapy, more than half of the patients relapse locoregional or metastatic ${ }^{[2]}$. With chemotherapy and anti-epidermal growth factor receptor, advanced treatment was relatively limited before the arrival of immunotherapy in

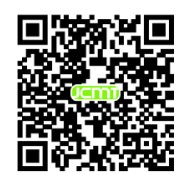


the therapeutic arsenal. Head and neck cancers are cancers inducing immunosuppression and therefore particularly likely to respond well to immunotherapy. The purpose of this article is to review studies validating the use of first-line and second-line checkpoint inhibitors (CPIs) in HNSCC, as well as studies evaluating new indications. The aim is also to evaluate the new immunotherapy modalities under evaluation and to discuss the difficulties of using these new treatment.

\section{VALIDATED TREATMENT IN SECOND LINE}

Nivolumab was the first molecule to be approved by the Food and Drug Administration with the Checkmate 141. This showed an overall survival improvement of 7.7 months vs. 5.1 months $(\mathrm{HR}=0.68$, 95\%CI: 0.54-0.86) compared with monotherapy with docetaxel, methotrexate or cetuximab in HNSCC progressing after a first platinum-based line. This difference was greater in PD-L1 patients $>1 \%$ with an OS of 8.2 months vs. 4.7 months, but the curves were also separated for PD-L1 negative patients (<1\%). Nivolumab was therefore approved regardless of PD-L1 expression ${ }^{[3]}$.

Pembrolizumab was studied in the same situation with the Keynote 0-40 Phase III study, but this time docetaxel was given every 3 weeks at a dose of $75 \mathrm{mg} / \mathrm{m}^{2}$. While the first analysis was not significant, the discount reached statistical significance with a median overall median survival of 8.4 months (95\%CI: 6.4-9.4) vs. 6.9 months (95\%CI: 5.9-8.0), $\mathrm{HR}=0.80$ (95\% CI: $0.65-0.98, P=0.0161$ ). The reduction in HR relative to nivolumab can be explained by several factors. First, a cross-over effect in the chemotherapy group with many patients receiving immunotherapy secondarily, secondly because a majority of patients received docetaxel, which is the most effective chemotherapy and also by the use of docetaxel every three weeks used in this study which is probably more effective than the weekly rhythm used with nivolumab. In the subgroup analyzes, survival was significantly increased from 7.9 to 11.6 months in favor of pembrolizumab in patients with $\mathrm{PD}-\mathrm{L} 1>50 \%$. There was no difference in the $\mathrm{PD}-\mathrm{L} 1$ population $<50 \%$. These results led to the approval in Europe of pembrolizumab in PD-L1 patients $>50 \%{ }^{[4]}$.

A third phase 3 trial, the EAGLE study, compared an anti-PD-L1 (durvalumab) alone or with an antiCTLA4 (Tremelimumab) to a standard chemotherapy. This study was negative for the overall survival, but the durvalumab arm as monotherapy was consistent with nivolumab and pembrolizumab with a median overall survival at 7.6 months and a response rate of $17.9 \%$. In addition, the control arm was superior to what was expected because weekly paclitaxel was predominantly used (which is probably the best treatment option for these patients) and there was an important crossover with control arm patients receiving immunotherapy ${ }^{[5]}$.

\section{FIRST LINE TREATMENT}

The Keynote 048 trial is an open-label, randomized, phase 3 trial comparing 887 patients in first line with pembrolizumab to the extreme protocol [cisplatin or carboplatin, 5 -fluorouracile (5FU) and cetuximab] ${ }^{[6]}$. Pembrolizumab was administered at a fixed dose of $200 \mathrm{mg}$ every 3 weeks alone or in combination with cisplatin and 5FU chemotherapy. The stratification factors were the expression of PD-L1 by tumor proportion score (TPS: expression of PD-L1 by tumor cells $\geq 50 \% v s .<50 \%$ ), p16 status for oropharyngeal tumors (positive vs. negative) and Eastern Cooperative Oncology Group status (0 vs. 1). Pembrolizumab showed a benefit in overall survival over chemotherapy and cetuximab in the combined positive score (CPS) population $\geq 20$ (a score that studies expression on both tumor cells and immune cells in the microenvironment) with a median of 14,8 months $v s .11$ months; HR 0.58 (95\%CI: 0.44-0.78). This advantage was found in the group of CPS $\geq 1$ with a median of 12.3 months $v s .10 .4$ months; HR 0.74 (95\%CI: 0.61-0.90). But it is important to note that if survival is better with pembrolizumab, at the beginning immunotherapy is deleterious for some patients with more deaths in the first six months. In terms of tolerance, treatment-related adverse event levels of Gr 3-5 were 17\% with pembrolizumab against $69 \%$ with 


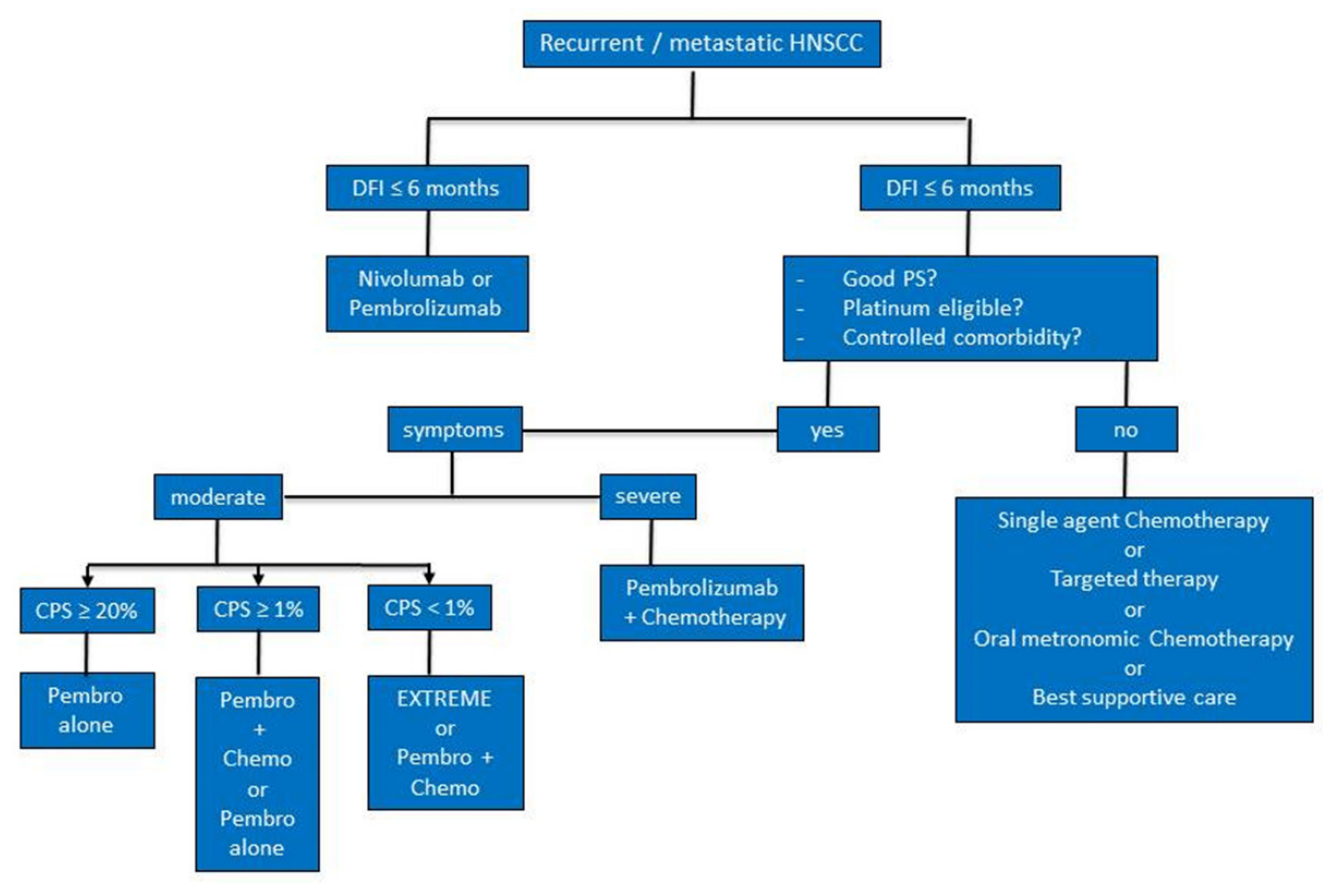

Figure 1. Proposed therapeutic decision tree in the recurrent/metastatic head and neck squamous cell carcinoma (HNSCC)

the Extreme protocol. The update during the American Society of Clinical Oncology (ASCO) 2019 also showed a benefit in overall survival in the pembrolizumab group associated with chemotherapy compared with the Extreme group, regardless of the subgroup of patients. The median OS was 14.7 months vs. 11 months, respectively; HR 0.60 (95\%CI: $0.45-0.82)$ for the CPS group $\geq 20,13.6$ months vs. 10.4 months; HR 0.65 (95\%CI: 0.53-0.80) for the CPS group $\geq 1$ and 13 vs. 10.7; HR 0.72 (95\%CI: 0.60-0.87) months in total population.

It is therefore the first randomized trial that demonstrates an improvement in overall survival compared to standard platinum and cetuximab treatment in the first line. This trial also establishes the CPS as a valid marker for selecting patients most likely to benefit from pembrolizumab monotherapy. In CPS $\geq$ 20 , the toxicity profile favors treatment with pembrolizumab monotherapy, whereas the combination of pembrolizumab with chemotherapy seems the best alternative for CPS $<20$ that can benefit. Pembrolizumab in monotherapy may also be discussed in slowly progressive patients with CPS $<20$. Be that as it may, it is likely that this study will upset the frontline management of HNSCC cancers in the front line.

There are currently two other studies currently underway evaluating first-line CPIs. Durvalumab alone or in combination with tremelimumab is compared to the EXTREME protocol in the KETREL trial and the nivolumab and ipilimumab combination is still compared to the same protocol in the CA609-651 trial.

Finally, these new studies will change the management of HNSCC cancers. We propose in Figure 1 a decisional tree for the treatment of recurrent or metastatic HNSCC.

\section{IMMUNOTHERAPY MORE PRECOCIOUS}

Because of the improvement in overall survival provided by the inhibitors of chekpoint, the question arises of introducing these treatments earlier in curative intent for localized disease, in neoadjuvant or adjuvant treatment. 
Table 1. Recent immunotherapy approaches in head and neck squamous cell carcinoma

\begin{tabular}{|c|c|c|c|}
\hline Immunotherapy & Mecanism of action & $\begin{array}{c}\text { Stage of clinical } \\
\text { develoment }\end{array}$ & Setting \\
\hline M7824 & $\begin{array}{l}\text { Bispecific anti-PD-L1 and anti-TGF- } \beta \\
\text { antibody }\end{array}$ & Phase I & $\begin{array}{l}\text { Recurrent/metastatic HNSCC in second } \\
\text { therapeutic line }\end{array}$ \\
\hline Monalizumab with cetuximab & $\begin{array}{l}\text { New checkpoint inhibitor targeting } \\
\text { NKG2A + anti EGFR }\end{array}$ & Phase II & Recurrent/metastatic HNSCC \\
\hline Danvatirsen with durvalumab & $\begin{array}{l}\text { Inhibitory antisense oligonucleotide } \\
\text { of STAT3 + anti-PD-L1 }\end{array}$ & Phase Ib/II & Metastatic HNSCC naive of anti-PD-L1 \\
\hline SD101 with pembrolizumab & TLR9 agonist + anti-PD-1 & Phase I/II & $\begin{array}{l}\text { Recurrent/metastatic HNSCC, anti-PD1 } \\
\text { naive }\end{array}$ \\
\hline Epacadostat & Oral ID01 inhibitor & Phase III failures & Recurrent/metastatic HNSCC \\
\hline Lenvatinib & Broad-spectrum TKI & Phase Ib/II & $\begin{array}{l}\text { Metastatic HNSCC, after at least one } \\
\text { line of chemotherapy }\end{array}$ \\
\hline MEDI6974 with durvalumab & Ox40 agonist antibody + anti-PD-L1 & Phase Ib & Before surgery with stage III-IVA HNSCC \\
\hline $\begin{array}{l}\text { Dendritic cell vaccination with } \\
\text { conventional chemotherapy }\end{array}$ & $\begin{array}{l}\text { Mature CD loaded with WT1 } \\
\text { peptides + OK-432 (TLR-4 ligand) }\end{array}$ & Phase I/II & Recurrent/metastatic HNSCC \\
\hline
\end{tabular}

HNSCC: head and neck squamous cell carcinoma; EGFR: epidermal growth factor receptor; TGF: transforming growth factor

In adjuvant, trials are in progress with comparative immunotherapies or added to cisplatin or cetuximab during radiotherapy, but there is currently no efficacy data. There is no sign of toxicity so we expect a better tolerance than current treatments. A randomized Phase II trial compared potentiated radiotherapy with cetuximab or pembrolizumab in inoperable patients not eligible for cisplatin. Tolerance was better in the pembrolizumab group with a 3-4 grade toxicity of $69 \%$ vs. $88 \%$ with cetuximab. There was particularly less mucositis and radiodermatitis with pembrolizumab ${ }^{[7]}$.

In neoadjuvant, a phase I is in progress that combines durvalumab with TPF (cisplatin docetaxel 5FU) to potentiate induction chemotherapy.

A phase 2 study also studied still in neoadjuvant 23 patients with high-risk HNSCC cancer (probable R1 resection or capsular rupture) who received pembrolizumab 1 to 3 weeks before surgery. There was $47 \%$ response with an anti-tumor effect on more than $10 \%$ of the surface, including $32 \%$ major response on more than $70 \%$ of the surface and 1 complete response ${ }^{[8]}$. Similar results have been observed with nivolumab ${ }^{[9]}$.

It is also possible that immunotherapy may make subsequent chemotherapy more effective, which would favor earlier administration of immunotherapy.

\section{NEWS IMMUNOTHERAPY}

Many immunotherapy molecules with different mechanisms of action are currently under development. A review of the 2016 literature presented the latest insights into the field of immunotherapy for HNSCC ${ }^{[10]}$. Many immunotherapy associations were already being tested. The goal here is to present some of these innovations with a promising future to show the diversity of this one. Table 1 summarizes the molecules presented.

A phase 1 study showed promising results with a bispecific anti-PD-L1 and anti-transforming growth factor- $\beta$ (TGF- $\beta$ ) antibody (M7824). The goal is to inhibit TGF- $\beta$, which increases tumor immunodepression. In addition, inhibition of the TGF- $\beta$ pathway could increase the activity of antiPD-L1. Among 32 patients, 7 had partial response, 6 had tumor stability, and grade III was found among 10 patients (31.3\%). Further development will be interesting to follow ${ }^{[1]]}$.

Monalizumab is a new CPI targeting NKG2A. This is expressed on CD8+ lymphocytes and on NK lymphocytes. The ligand of NKG2A, human leucocyte antigens (HLA)-E, is overexpressed in HNSCC. 
Blocking this interaction stimulate CD8+ and NK lymphocytes and increase antibody-dependent cellmediated cytotoxicity (ADCC). Since Cetuximab acts mainly via an ADCC mechanism, it could be potentiated by Monalizumab. A phase II study included 40 patients to receive weekly cetuximab combined to monalizumab every two weeks after failur of platin. The response rate, which was the primary endpoint, was $27.8 \%$, or 11 out of 40 patients (compared to $13 \%$ with cetuximab alone according to historical data). The tolerance was particularly good, making this association a promising issue ${ }^{[12]}$. It is interesting to note that monalizumab seems to be effective after failure of an anti-PD-1.

Another phase Ib/II study investigated the combination of danvatirsen with durvalumab among patients with metastatic HNSCC naive of anti-PD-L1. Danvatirsen is an inhibitory antisense oligonucleotide of STAT3 (that stimulates the immunosuppressive function of the microenvironment). Among 44 treated patients, $23 \%$ of responses (higher than those found with durvalumab monotherapy) were found with 3 complete responses. An increase of transaminases and transient thrombocytopenia were the major toxicities and it seems there is no additive toxicity of the two treatments ${ }^{[13]}$.

SD101 is a TLR9 agonist stimulating the activity of dendritic cells. This later became antigen-presenting cells to activate an anti-tumor response via T lymphocytes. A phase I/II study investigated the combination with pembrolizumab. Patients received pembrolizumab every 3 weeks and SD-101 injected into a single tumor site each week for 4 injections and then every 3 weeks up to 7 injections. Among the 26 patients included, the median injections of SD-101 administered were 5. Patients experienced injection site reactions and influenza-like illness with $28 \%$ of toxicities $\geq$ grade 3 . The immune-induced effects of pembrolizumab do not appear to have increased. The response rate in mITT (that is to say, among the 23 patients who had reached at least the first tumor evaluation) was $30.4 \%$ with 7 partial responses, 3 stabilities, 10 progressions in imaging and 3 clinical progressions before the scan. Tumor size reductions $\geq 30 \%$ were observed in injected lesions (8/18 or $44.4 \%$ ) as well as in non-injected lesions (8/14 or 57.1\%). At the time of the analysis, 5 of the 7 responses were ongoing ( 1 to 27 weeks). Therefore, stimulation of dendritic cells seems promising with activity in the injected site, but also at a distance, at the cost however of a significant toxicity ${ }^{[14]}$.

Epacadostat is an oral IDO1 inhibitor (indoleamine 2,3-dioxygenase) showing good phase I and II in combination with pembrolizumab ${ }^{[15]}$. IDO1 is an enzyme expressed in the tumor microenvironment that has an immunosuppressive effect by decreasing tumor infiltration by cytotoxic T lymphocytes (TILs). But in the face of phase III failures in melanoma, development has unfortunately been halted ${ }^{[15]}$.

Associations with tyrosine kinase inhibitors (TKIs) have also been studied in a phase Ib/II study. Lenvatinib, a broad-spectrum TKI, has been associated with pembrolizumab in 22 progressive metastatic patients after at least one line of chemotherapy. The 24 -week response rate was $36 \%$ with a median response time of 8.2 months, a progression-free survival (PFS) of 8.2 months, and one year progression-free survival rate of $37 \%$. But the toxicity is important with $91 \%$ of side effect, of which $14 \%$ of grade 4 and $18 \%$ of patients who had to stop the treatment. The main symptoms were fatigue, loss of appetite, hypertension and digestive disorders. Associations with other less toxic tyrosine kinase inhibitors may be considered ${ }^{[16]}$.

Another trial associated durvalumab with an activating agonist of the immune response, MEDI6974 (Ox40 agonist antibody). The treatments were administered for a short time before surgery. An increase and activation of cytotoxic lymphocytes within the tumor has been demonstrated. This association could potentially be interesting ${ }^{[17]}$.

A phase I/II study investigated dendritic cell vaccination with conventional chemotherapy in patients with head and neck cancer ${ }^{[18]}$. Eleven patients with relapsed or refractory disease were recruited (with HLA-A2 or HLA-A24 positive as well as WT1 expression, a tumor antigen). Mature dendritic cell (DC) loaded 
with WT1 peptides were injected intradermally approximately every 2-3 weeks. OK-432 (TLR-4 ligand) was administered subcutaneously near the vaccination sites to activate the DCs. Treatment was generally well tolerated with none of the patients with grade 2 adverse events except for hematologic adverse events with leucocytopenia and grade 2 anemia. Non-hematologic adverse events were consistent with the classic adverse effects of chemotherapy (discomfort, nausea, anorexia, and constipation except transient erythema at injection sites and low-grade fever). 5 patients presented a stabilization of the disease and 6 patients a progression of the disease. The median PFS and OS were 6.4 months and 12.1 months, respectively. But the median duration of PFS and OS of patients with stable disease was significantly longer than that of patients with progressive disease. The median PFS was 13.0 months vs. 2.8 months $(P=0.00136)$ and the median OS was 30.3 months $v$ s. 8.1 months $(P=0.0126)$. Two patients had a long-term survival of $30.3 \mathrm{~m}$ and $44.4 \mathrm{~m}$. Furthermore, all patients with stable disease showed improvement of WT1-specific immune responses after vaccination in all tests performed [HLA tetramer, interferon gamma (IFN $\gamma$ ) ELISPOT and mobilization CD107a] as well as an increased Th1/Th2 ratio of 1.36 times on average. Dendritic cell vaccination is therefore an interesting research path because of its efficacy in some patients and its good tolerance ${ }^{[18]}$.

In conclusion, many promising new immunotherapies are being developed with different mechanism of action. Some may be used alone and other combined with CPIs or other conventional therapy.

\section{DIFFICULTY OF ASSESSING IMMUNOTHERAPY}

Evaluation of immunotherapies may be different and more difficult than conventional anti-tumor treatments. In fact, patients treated with CPI observed phenomena of pseudo-progression (tumor response after an initial progression) and, on the contrary, hyperprogression phenomena with a rapid and significant increase in tumor volume.

These pseudo progression are probably due to infiltration of immune and inflammatory cells and/ or a delayed clinical response. These phenomena are relatively rare but may explain the benefit of immunotherapy treatment beyond progression. For example, in the Checkmate 141 study, of 240 patients randomized to receive nivolumab, 146 showed progression according to RECIST. Sixty-two of these patients continued nivolumab and 84 discontinued treatment. Of the 60 patients who continued nivolumab evaluable for the response, 15 (25\%) had not changed their tumor burden and 15 (25\%) had a reduction in the size of the target lesion ( $3 \%$ of patients had responded by RECIST after progression). This attests the interest to continue the treatment beyond the progression if the general state remains correct and in the absence of rapid progression. Note that the expression of PDL1 does not seem to differentiate patients who will be treated or not beyond progression ${ }^{[19]}$.

This pseudo progression can in part explain the possible increase of efficacy of subsequent chemotherapy after failure of anti-PD-1. Indeed a French retrospective study in 82 patients receiving chemotherapy (45\% monotherapy and 55\% combination therapy) after failure of immunotherapy showed a $30 \%$ responses and a 57\% disease control, higher than those usually observed in the 3rd line. Chemotherapy could also activate the immune system by releasing tumor antigens, with check point inhibitors known to remain in the body for a long time.

Hyperprogressions are associated with poorer survival. A French retrospective study suggested that hyperprogression can be observed in $29 \%$ of patients treated by immunotherapy ${ }^{[20]}$. This controversial phenomenon could be induced by an excessive immune reaction, a paradoxical action of anti-PD-1/PD-L1 promoting tumor development, or ultimately only the natural history of advanced phase cancer.

Finally, unlike conventional chemotherapy, the occurrence of autoimmune adverse events seems to correlate with the effectiveness of the CPIs. In an analysis of 114 patients treated with anti-PD-1: 43\% exhibited 
immunological toxicity. These patients had higher response rates (30.6\% vs. $12.3 \%)$, better progressionfree survival (6.9 months vs. 2.1 months) and improved overall survival (12.5 months vs. 6.8 months). This analysis may of course be biased since patients who benefit from immunotherapy pursue it longer and are therefore more likely to develop immunological toxicity ${ }^{[2]}$.

\section{PREDICTIVE FACTOR OF EFFICACY OF IMMUNOTHERAPY}

One of the major challenges of immunotherapy is to select patients responding to immunotherapies with predictive biomarkers of effectiveness. Currently, the only one used is the expression of PDL1 but it is very imperfect, especially with excellent results in PDL1 negative patients.

The expression of PD-L2 and the signature IFN $\gamma$ have been explored, but do not seem particularly convincing.

HNSCC have been classified into 6 groups: immunoreactive, inflammatory, Human Papilloma Virus (HPV)-like, classical, hypoxemic and mesenchymal ${ }^{[2]}$. The inflammatory and immunoreactive profiles are probably more sensitive to immunotherapies because they are strongly infiltrated by cells of the immune system. The important tumoral infiltration by the CD8 lymphocytes could characterize this inflammatory type.

In a cohort of 34 patients with significan CD8+ lymphocyte infiltration and a high PD-1/TIM3 expression ratio (regardless of HPV status and smoking status) showed an increase in overall survival (84 months vs. 13 months) with anti-PD-1 treatment, compared with non-inflammatory tumors ${ }^{[23]}$.

The estimation of the mutational load (TMB) in the primary tumor, but also from the circulating DNA could also become a predictive biomarker ${ }^{[24]}$.

There is also evidence that HPV-positive tumors may be better able to respond to anti-PD1. There is indeed a biological rationale for better efficacy of anti-PD1 in HPV + tumors since they show more immune infiltrates and more markers of activated T cells. A retrospective analysis of 54 patients treated after platinum failure with pembrolizumab (32 patients) or nivolumab (22) overall survival was significantly better for HPV positive patients: 17 months $v s .4 .5$ months. Similarly, the duration of anti-PD1 treatment was significantly increased in positive HPV: 7 months vs. 3 months ${ }^{[25]}$.

Much simpler, a study presented at ASCO 2018 has recently been shown to have a high lymphocyte neutrophil count associated with tumor inflammation and poor prognosis ${ }^{[26]}$. The 114 patients were treated with anti-PD1 classified in 4 quartiles according to this ratio. Patients with the lowest ratio had an overall survival well above 12.5 months compared to those with the highest ratio $(4.1$ months, $P<0.0001)$. In this cohort, PD-L1 expression did not discriminate patients. Thus, a very simple and clinical marker proves to be a powerful prognostic factor for the efficacy of immunotherapies.

\section{CONCLUSION}

Squamous cell carcinoma of the head and neck has not been spared by the revolution of immunotherapy. Indeed, the CPIs are now an integral part of the therapeutic arsenal and new indications are emerging either as monotherapy or in combination. In addition, many other classes of molecules are currently under development. The selection of patients who will benefit most from immunotherapy with new biomarkers remains an important challenge in the use of these new therapies. The question of the optimal duration and determination of the best combinations of these immunotherapy treatments remains unresolved. The therapeutic management of HNSCC cancers will continue to be disrupted in the coming years. 


\section{DECLARATIONS}

\section{Authors' contributions}

Wrote and reviewed the manuscript: Gauduchon T, Reverdy T, Gau M, Karabajakian A, Collet L, Neidhardt EM, Fayette J

\section{Availability of data and materials}

Not applicable.

\section{Financial support and sponsorship}

None.

\section{Conflicts of interest}

All authors declared that there are no conflicts of interest.

\section{Ethical approval and consent to participate}

Not applicable.

\section{Consent for publication}

Not applicable.

\section{Copyright}

(C) The Author(s) 2019.

\section{REFERENCES}

1. Bray F, Ferlay J, Soerjomataram I, Siegel RL, Torre LA, et al. Global cancer statistics 2018: GLOBOCAN estimates of incidence and mortality worldwide for 36 cancers in 185 countries. CA Cancer J Clin 2018;68:394-424.

2. Karabajakian A, Toussaint P, Neidhardt EM, Paulus V, Saintigny P, et al. Chemotherapy for recurrent/metastatic head and neck cancers. Anticancer Drugs 2017;28:357-61.

3. Ferris RL, Blumenschein G, Fayette J, Guigay J, Colevas AD, et al. Nivolumab vs investigator's choice in recurrent or metastatic squamous cell carcinoma of the head and neck: 2-year long-term survival update of CheckMate 141 with analyses by tumor PD-L1 expression. Oral Oncol 2018;81:45-51.

4. Cohen EEW, Soulières D, Le Tourneau C, Dinis J, Licitra L, et al. Pembrolizumab versus methotrexate, docetaxel, or cetuximab for recurrent or metastatic head-and-neck squamous cell carcinoma (KEYNOTE-040): a randomised, open-label, phase 3 study. Lancet 2019;393:156-67.

5. Ferris RL, Even C, Haddad R, Tahara M, Goswami T, et al. Phase III, randomized, open-label study of durvalumab (MEDI4736) monotherapy, or durvalumab + tremelimumab, versus standard of care $(\mathrm{SoC})$, in recurrent or metastatic $(\mathrm{R} / \mathrm{M})$ squamous cell carcinoma of the head and neck (SCCHN): eagle. J Immunother Cancer 2015;3:P150.

6. Burtness B, Harrington KJ, Greil R, Soulières D, Tahara M, et al. KEYNOTE-048: Phase 3 study of first-line pembrolizumab (P) for recurrent/metastatic head and neck squamous cell carcinoma (R/M HNSCC). Available from: https://oncologypro.esmo.org/MeetingResources/ESMO-2018-Congress/KEYNOTE-048-Phase-3-study-of-first-line-pembrolizumab-P-for-recurrent-metastatic-head-andneck-squamous-cell-carcinoma-R-M-HNSCC. [Accessed on 23 Oct 2019]

7. Sun XS, Sire C, Tao Y, Martin L, Alfonsi M, et al. A phase II randomized trial of pembrolizumab versus cetuximab, concomitant with radiotherapy (RT) in locally advanced (LA) squamous cell carcinoma of the head and neck (SCCHN): first results of the GORTEC 2015-01 "PembroRad" trial. J Clin Oncol 2018;36:6018.

8. Uppaluri R, Zolkind P, Lin T, Nussenbaum B, Jackson RS, et al. Neoadjuvant pembrolizumab in surgically resectable, locally advanced HPV negative head and neck squamous cell carcinoma (HNSCC). J Clin Oncol 2017;35:6012.

9. Ferris RL, Gonçalves A, Baxi SS, Martens U, Gauthier H, et al. LBA46An open-label, multicohort, phase 1/2 study in patients with virus-associated cancers (CheckMate 358): safety and efficacy of neoadjuvant nivolumab in squamous cell carcinoma of the head and neck (SCCHN). Ann Oncol 2017;28.

10. Economopoulou P, Kotsantis I, Psyrri A. The promise of immunotherapy in head and neck squamous cell carcinoma: combinatorial immunotherapy approaches. ESMO Open 2016;1:e000122.

11. Cho BC, Daste A, Ravaud A, Salas S, Isambert N, et al. 1048OM7824 (MSB0011359C), a bifunctional fusion protein targeting PD-L1 and TGF- $\beta$, in patients (pts) with advanced SCCHN: results from a phase I cohort. Ann Oncol 2018;29.

12. André P, Denis C, Soulas C, Bourbon-Caillet C, Lopez J, et al. Anti-NKG2A mAb is a Checkpoint Inhibitor that Promotes Anti-tumor 
Immunity by Unleashing Both T and NK Cells. Cell 2018;175:1731-43.e13.

13. Cohen EEW, Harrington KJ, Hong DS, Mesia R, Brana I, et al. 1044OA phase Ib/II study (SCORES) of durvalumab (D) plus danvatirsen (DAN; AZD9150) or AZD5069 (CX2i) in advanced solid malignancies and recurrent/metastatic head and neck squamous cell carcinoma (RM-HNSCC): Updated results. Ann Oncol 2018;29:mdy287.

14. Cohen EEW, Nabell L, Wong DJL, Day TA, Daniels GA, et al. Phase 1b/2, open label, multicenter study of intratumoral SD-101 in combination with pembrolizumab in anti-PD-1 treatment naïve patients with recurrent or metastatic head and neck squamous cell carcinoma (HNSCC). J Clin Oncol 2019;37:6039.

15. Mitchell TC, Hamid O, Smith DC, Bauer TM, Wasser JS, et al. Epacadostat plus Pembrolizumab in patients with advanced solid tumors: phase I results from a multicenter, open-label phase I/II trial (ECHO-202/KEYNOTE-037). J Clin Oncol 2018:JCO2018789602.

16. Taylor MH, Rasco DW, Brose MS, Vogelzang NJ, Richey SL, et al. A phase 1b/2 trial of lenvatinib plus pembrolizumab in patients with squamous cell carcinoma of the head and neck. J Clin Oncol 2018;36:6016.

17. Bell RB, Duhen R, Leidner RS, Curti BD, Ballesteros-Merino C, et al. Neoadjuvant anti-OX40 (MEDI6469) prior to surgery in head and neck squamous cell carcinoma. J Clin Oncol 2018;36:6011.

18. Ogasawara M, Miyashita M, Yamagishi Y, Ota S. Phase I/II pilot study of Wilms' tumor 1 peptide-pulsed dendritic cell vaccination combined with conventional chemotherapy in patients with head and neck cancer. Ther Apher Dial 2019;23:279-88.

19. Haddad R, Concha-Benavente F, Blumenschein G, Fayette J, Guigay J, et al. Nivolumab treatment beyond RECIST-defined progression in recurrent or metastatic squamous cell carcinoma of the head and neck in CheckMate 141: a subgroup analysis of a randomized phase 3 clinical trial. Cancer 2019;125:3208-18.

20. Saâda-Bouzid E, Defaucheux C, Karabajakian A, Palomar Coloma V, Servois V, et al. Hyperprogression during anti-PD-1/PD-L1 therapy in patients with recurrent and/or metastatic head and neck squamous cell carcinoma. Ann Oncol 2017;28:1605-11.

21. Foster CC, Kochanny S, Khattri A, Acharya R, Dekker A, et al. Association of immune-related adverse events (irAEs) with improved response, progression-free survival, and overall survival for patients with metastatic head and neck cancer receiving anti-PD-1 therapy. J Clin Oncol 2018;36:6014.

22. De Cecco L, Nicolau M, Giannoccaro M, Daidone MG, Bossi P, et al. Head and neck cancer subtypes with biological and clinical relevance: meta-analysis of gene-expression data. Oncotarget 2015;6:9627-42.

23. Hanna GJ, Liu H, Jones RE, Bacay AF, Lizotte PH, et al. Defining an inflamed tumor immunophenotype in recurrent, metastatic squamous cell carcinoma of the head and neck. Oral Oncol 2017;67:61-9.

24. Khagi Y, Goodman AM, Daniels GA, Patel SP, Sacco AG, et al. Hypermutated circulating tumor DNA: correlation with response to checkpoint inhibitor-based immunotherapy. Clin Cancer Res 2017;23:5729-36.

25. Kirtane K, Muzaffar J, Slebos R, Chung CH. Association between human papillomavirus (HPV) status and duration of response of anti-programmed cell death protein-1 (PD-1) inhibitors in patients with recurrent/metastatic (R/M) head and neck squamous cell carcinoma (HNSCC). J Clin Oncol 2019;37:6044.

26. Foster CC, Kochanny S, Khattri A, Acharya R, Dekker A, et al. Association of a baseline neutrophil-to-lymphocyte ratio (NLR) with progression-free and overall survival in head and neck cancer patients receiving anti-PD-1 therapy. J Clin Oncol 2018;36:6038. 